\title{
Fangchinoline Inhibits Breast Tumor Proliferation and Induces Apoptosis in MDA-MB-231 Cell Line in Vivo
}

\author{
Deng $\mathrm{X}^{1 \#}$, Liu $\mathrm{G}^{1 \#}$, Hu N $\mathrm{N}^{2}$, Yuan $\mathrm{C}^{3}$, Liu $\mathrm{Z}^{4}$, Li F $\mathrm{F}^{1}$, Pan $\mathrm{J}^{5}$ and Wang $\mathrm{C}^{* 1}$
}

${ }^{1}$ Department of Biochemistry and Molecular Biology, ChongQing Medical University, China

${ }^{2}$ Department of Orthopaedic Surgery, The First Affiliated Hospital of Chongqing Medical University, Chongqing, China

${ }^{3}$ Department of Biochemistry \& Molecular Biology, College of Medical Science, China Three Gorges University, Yichang, HuBei, China

${ }^{4}$ Department of Orthopaedics, The Fourth Affiliated Hospital of China Medical University, Shenyang, China ${ }^{5}$ Department of Biomedical and Chemical Engineering, Syracuse University, Syracuse, New York, United States of America

\# The two authors contribute equally to this work.

${ }^{*}$ Corresponding author: Wang C, Department of Biochemistry and Molecular Biology, ChongQing Medical University, 1\# Yixueyuan Road, Yuzhong District, ChongQing 400016, China, E-mail: cdwhust@163.com

Citation: Deng X, Liu G, Hu N, Yuan C, Liu Z, et al. (2015) Fangchinoline Inhibits Breast Tumor Proliferation and Induces Apoptosis in MDA-MB-231 Cell Line in Vivo. J Cancer Sci Clin Oncol 2(2): 201. doi: $10.15744 / 2394-6520.2 .201$

Received Date: January 20, 2015 Accepted Date: June 08, 2015 Published Date: June 10, 2015

\begin{abstract}
Objective: This study was purposed to investigate the effect of Fangchinoline (Fan) on the regulation of tumor growth and apoptosis in vivo.

Methods: Immunohistochemistry (IHC) was used to determine the protein levels of p27, CD117 and ki67 in tumor tissue of xenograft. After treatment of Fan, the morphologic changes were observed by DAPI staining, Hoechst staining and TUNEL detection under fluorescent microscopy. The protein expression of Bax, Bcl-2 active Caspase- 3 and cytochrome-c were measured by Western blotting.

Results: Fan repressed xenograft tumor growth in vivo by $33 \%$ compared with control and inhibited tumor cells proliferation by upregulating p27 and down-regulating CD117 and Ki67. DAPI staining, Hoechst staining and TUNEL staining showed that Fan induced apoptosis in vivo. Furthermore, the protein levels of Bcl-2 decreased, while the expression of Bax and active Caspase-3 and cytochrome-c increased.

Conclusion: Fan showed significant effect of down-regulation of CD117 and ki67. It also could up-regulation of p27 and induce apoptosis in vivo. The mechanisms may involve up-regulating $\mathrm{Bax} / \mathrm{Bcl}-2$ ratio and expression of active Caspase-3 and cytochrome-c.

Keywords: Fangchinoline; Breast Tumor; Apoptosis
\end{abstract}

\section{Introduction}

Radix Stephaniae tetrandrae, dry roots of Stephaniae tetrandrine S. Moore (Menispermaceae), is officially and traditionally used as an analgesic and anti-hypertension drug in China. The main chemical constituents in radix Stephania tetrandrae are tetrandrine (Tet) and fangchinoline (Fan) [1]. Tet has an antiadherent effect that might result from its inhibition of $\mathrm{Ca}^{2+}$ influx and reactive oxygen species formation, resulting in suppression of up-regulation of Mac-1 and, in turn, neutrophil adhesion to fibrinogen [2]. In the past several years, important progress had been made in the study of the mechanisms of effect of Fan on some diseases. Lin Ty et al. reported that Fan inhibited glutamate release from rat cerebral cortex nerve terminals and had been shown to possess neuroprotective properties [3]. Fan has anti- inflammatory effects [4] and antioxidant activity [5]. Most recently, Zhang YH et al. reported that Fan inhibits proliferation and cells cycle progression of aortic vascular smooth muscle cell through inhibition of ERK1/2 activation and c-fos expression [6]. However, the anti-tumor activity of Fan in breast cancer is still unknown.

Breast cancer remains the second leading cause of cancer deaths among women. The multifactorial nature of breast carcinogenesis, as well as the cellular and molecular diversity within tumors, have rendered it difficult for the development of molecularly targeted treatments and the drug may be applied to the broad range of breast tumors. Nerve growth factor has been used as a target in curing breast cancer in the form of anti-NGF antibodies or small interfering RNA against NGF displaying inhibition of tumor growth and metastasis [7]. Nanobin encapsulation of $\mathrm{As}_{2} \mathrm{O}_{3}$ improves the pharmacokinetics and antitumor efficacy in breast cancer in vivo $[8,9]$. Based on the progress of anti-tumor therapeutics, we selected Fan, an efficient component of Stephaniae tetrandrine to test its potential effect on breast cancer. 
We hypothesize that Fan induces breast cancer apoptosis in MDA-MB-231 breast cancer cell lines in vivo. Moreover, our data shows that Fan repressed the tumor growth. In TUNEL experiment, the positive cell immunofluorescence of the cells treated by Fan was much more than that of the control. Immunohistochemistry demonstrated that CD117 and Ki67 protein expression levels were repressed by Fan. Moreover, western-blot showed that the protein expression levels of active caspase 3 and cytochrome-c were increased and ratio of Bax/Bcl-2 was also up-regulated by Fan.

\section{Material and Methods}

\section{Tumor xenografts in nude mice}

The experimental procedures were approved by our institutional animal research ethics committee with reference to the Chinese community guidelines for the use of experimental animals. Six-week-old female Balb/c-nu-nude mice were obtained from the Laboratory Animal Center of Hubei Province, China. The experimental license number is scxk (Hubei) 2003-0005. The mice were kept under sterile conditions and pathogen-free in isolated pathogen-free ventilation chambers at 20-22 and 45-50\% relative humidity. The animal rearing facility was maintained on a $12 \mathrm{~h}$ light-dark cycle. All the animals were given free access to sterilized food and water, and were under habituation for 10 days before experiment.

Cells suspension was obtained by trypsinization of confluent MDA-MB-231 cells. The mice were randomly assigned to control and various treatment groups of 12 mice each, and anesthetized with ip injection of $75 \mathrm{mg} / \mathrm{kg}$ ketamine and $10 \mathrm{mg} / \mathrm{kg}$ xylazine. The mice were treated with intravenous injection of Fan once daily (days 0-16). Cell suspension was injected subcutaneously into the back of each animal at a cell density of $1 \times 10^{7}$ in $200 \mu$ l of phosphate buffered saline. The day of tumor implantation was designated as day 0 . Tumor volume was measured using a digital caliper every 4 days, and was calculated as (length $\times$ width $\left.^{2}\right) / 2[10]$. The mice were monitored for 16 days after tumor inoculation. The body weight of all animals was recorded throughout the entire experimental period to assess drug toxicity. Any mortality during the course of the study was also recorded.

\section{Immunohistochemistry}

All immunostaining techniques were performed in paraffin-embedded 4 - $\mu \mathrm{m}$ tissue sections using a previous step of heat-induced antigen retrieval technique for all the antibodies. Thus, before incubation with the primary antibody, slides were treated in a microwave oven at $650 \mathrm{~W}$ for three cycles in a solution of $0.01 \mathrm{~mol} / \mathrm{l}$ sodium citrate ( $\mathrm{pH}$ 6). The following antibodies were used: anti-p27, anti-CD117, and anti-Ki67 (Santa Cruz Biotechnology Inc.). The sections were incubated with biotinylated immunoglobulin for $20 \mathrm{~min}$, followed by peroxidase-labeled streptavidin for 20 (Peroxidase Detection System, Novocastra Laboratories Ltd, Newcastle upon Tyne, UK) with diaminobenzidine chromogen as a substrate. Nuclei were counterstained with hematoxylin. In each experiment, positive and negative controls were included.

\section{DAPI, hoechst and TUNEL staining}

Tissue slides were wax depleted. The cells were stained with 6-diamidino-2-phenylindole (DAPI) or Hoechst- 3224 (Sigma) for 10 mins and then washed by PBS for 10 times. Slides treated by TNUEL reagent (Roche) for 60 min and then washed by PBS for three times and every time for $5 \mathrm{~min}$ each. Slides were mounted on glass slides for examination with fluorescence microscopy.

\section{Western blot analysis}

Tumor tissues were treated by liquid nitric and lysed with NP40 buffer (1\% NP-40, $0.15 \mathrm{M} \mathrm{NaCl}, 50 \mathrm{mM}$ Tris, $\mathrm{pH} 8.0)$ containing protease inhibitors (Sigma). Tissues lysates were centrifuged at $12,000 \mathrm{~g}$ for $10 \mathrm{mins}$ at $4{ }^{\circ} \mathrm{C}$, and the supernatants were stored at $-80^{\circ} \mathrm{C}$. Protein quantitation was performed with BCA protein assay reagent (Pierce, Rockford, IL, USA). Equal amounts of proteins from different groups were denatured in SDS sample buffer and separated on 10\% polyacrylamide-SDS gel based on the protein molecular weight. Proteins were transferred in $25 \mathrm{mM}$ of Tris, $192 \mathrm{mM}$ of glycine, and $20 \%$ methanol to polyvinylidene difluoride. Blots were blocked with Tris-buffered saline (TBS; $20 \mathrm{mM}$ of Tris- $\mathrm{HCl}$ [pH 7.5] and $137 \mathrm{mM}$ of $\mathrm{NaCl}$ ) containing 0.1\% Tween 20 and 5\% dried milk powder. Mouse monoclonal to Bax (Santa Cruze), Rabbit polyclonal to Bcl-2 (Santa Cruze), Rabbit polyclonal to cytochrome-c, p27 (Santa Cruze), Rabbit polyclonal antibody beta-actin (Santa Cruze) and Rabbit monoclonal to caspase 3 and active-caspase 3 (Santa Cruze) were used to detect the signals. The antigen-antibody complexes were visualized using the appropriate secondary antibodies (Sigma) and the enhanced chemiluminescence detection system, as recommended by the manufacturers. The proteins of interest were detected by using SuperSignal West Pico Chemiluminescent Substrate kit.

\section{Statistical analyses}

Statistical analysis was performed using SPSS-17.0 software. Data were analyzed using one-way analysis of variance and Tukey HSD test was applied as a post hoc test if significance was determined. Statistical significance for two groups was assessed using Student's t-test. The probability level $\mathrm{P}$ value of less than 0.05 was considered statistically significantly.

\section{Result}

\section{The anti-tumorgenic effect of Fan in nude mice xenograft}


To define whether Fan suppressed tumorigenic ability, we detected the tumor tissues volume every two days during the time of therapeutically using the Fan at $5 \mathrm{mg} / \mathrm{kg}$ in the nude mice. The volume of tumor in Fan treated group was smaller than that in the control group, as shown in Figure 1A. On the day of sacrifice (day 16), Fan treatments at the given dose resulted in about 50.5\% tumor suppression, and Fan-induced tumor tissues volume reduction was significantly from day 4 onwards, with average suppression of $33 \%$ (Figure 1B).

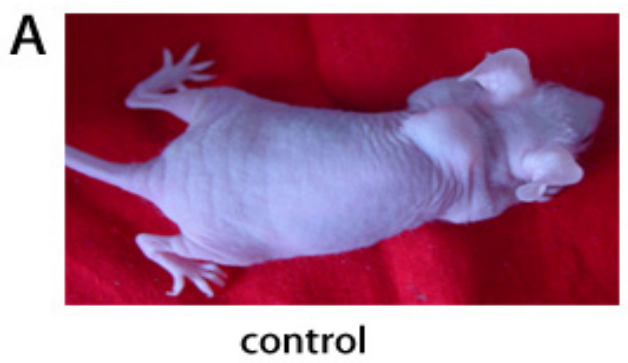

B
\begin{tabular}{|l|l|l|l|l|}
\hline Treament & Day4 & Day8 & Day12 & Day16 \\
\hline controll(mm $\left(m^{3}\right)$ & $112 \pm 16$ & $202.5 \pm 20$ & $378 \pm 18$ & $450 \pm 18$ \\
\hline $\begin{array}{l}\text { FAN }(5 m y / k g) \\
\left(\mathrm{mm}^{3}\right)\end{array}$ & $56 \pm 8^{*}$ & $141.75 \pm 20^{*}$ & $187.5 \pm 12.5^{* *}$ & $190 \pm 60^{* *}$ \\
\hline
\end{tabular}
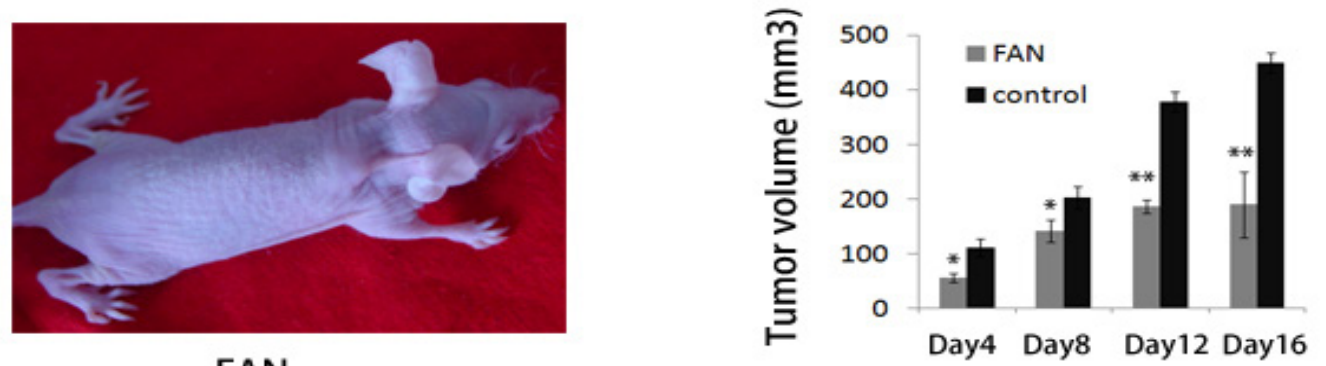

FAN

Figure 1: Fan inhibited tumor growth in MDA-MB-231 cells xenograft (A) Photographic illustration of tumors in control and Fan- treated nude mice on the day of sacrifice (16 days); (B) Quantitative analysis of tumor volume. The tumor volume of Fan $(5 \mathrm{mg} / \mathrm{kg})$ treated for 4 days was 0.5 times smaller than that of the control group $\left({ }^{*} \mathrm{p}<0.05 \mathrm{vs}\right.$ control). The tumor volume of Fan $(5 \mathrm{mg} / \mathrm{kg})$ treated for 8 days was 0.7 times smaller than that of the control group $\left({ }^{*} \mathrm{p}<0.05 \mathrm{vs}\right.$ control). The tumor volume of Fan $(5 \mathrm{mg} / \mathrm{kg})$ treated for 12 days was 0.5 times smaller than that of the control group $\left({ }^{*} \mathrm{p}<0.01 \mathrm{vs}\right.$ control). The tumor volume of Fan $(5 \mathrm{mg} / \mathrm{kg})$ treated for 16 days was 0.4 times smaller than that of the control group $\left({ }^{*} \mathrm{p}<0.01 \mathrm{vs}\right.$ control)

Fan up-regulated p27 and down-regulated CD117 and ki67 protein expression in nude mice xenograft

To characteristic of the effects of Fan on cell cycle, we studied whether p27 protein, the marker gene of cell cycle at the stage of G1, was regulated in tumor by using immunohistochemistry. As shown in Figure 2C and D, P27 gene expression was increased in the tumor treated by Fan in nude mice xenograft. To further testify the result, we detected the p27 protein expression in tumor using western-blotting. The western blot result showed that the p27 protein expression level was 4 times higher in tumor treated by Fan than that in the control tumor (Figure 4A, B). Histological H\&E staining analysis showed that the cell numbers of tumor treated by Fan is much less than that in the control group (Figure 2A, B). Furthermore, there are many empty places in the section of tumor treated by Fan (Figure 2A, B). We hypothesized that Fan inhibited cell proliferation in the tumor of nude mouse xenograft, and we detected CD117 and Ki67 gene expression, the related marker gene of cell proliferation in tumor which treated by Fan and the control group using immunohistochemistry. The immunohistochemistry results showed that stained by CD117 and Ki67 antibody, the positive cells in the Fan treated group are less than those in the control group. In addition, there are almost no positive cells stained by CD117 and Ki67 antibody in the tumor tissue treated by Fan (Figure 2E, F, G, H). The results indicated that Fan inhibited tumor cell proliferation through down-regulating CD117 and Ki67 protein expression in tumor growth.

\section{Fan induced apoptosis in nude mice xenograft}

To define if Fan induces cell apoptosis, we used DAPI staining to detect the nuclear of tumor cells. We detected characteristic apoptotic changes in the nuclei. The nuclei of tumor were condensed and fragmented in Fan treated tumor groups (Figure 3). In control groups, the nuclei of tumor showed no evidence of apoptosis (Figure 3 up. left panel). To confirm our finding that Fan induces tumor cell apoptosis, we used Hoechst staining of the tumor section. The results were the same as DAPI staining (Figure 3. middle, up and down). Furthermore, we used TUNEL staining to detect the apoptotic cell in tumor section. The results showed that there was no green fluorescence cell in the control group. However, there are many cells which displayed green fluorescence (Figure 3 right, down panel). Totally, all above three experiments confirmed that Fan induced cell apoptosis in tumor xenograft.

\section{Fan induced apoptosis through activating Caspase 3 and cytochrome-c and up-regulated the ratio of $\mathrm{Bax} / \mathrm{Bcl}-2$ in nude mice xenograft}

To gain further insight into the molecular mechanisms underlying Fan induced apoptosis, we analysis the ratio of Bax/Bcl-2, which is the key of apoptosis pathway. The result demonstrated that Fan induced Bax protein was increased 4 times and the Bcl2 protein was repressed about 1.8 times in the Fan treated group compared with the control group. The ratio of Bax/Bcl-2 was up-regulated about 8.2 times in Fan treated group compared with control group (Figure 4A, B). In addition, active-Caspase3 and 
cytochrome $\mathrm{C}$ are the two key proteins involved in mitochondrial apoptotic signal pathway. The western-blot results showed that the cytochrome-c level was 5 times higher in the Fan treated group than that in the control group, and active Caspase3 level was 4 times higher in the Fan treated group than that in the control group (Figure 4C and D). This suggested that Fan induced apoptosis in tumor xenograft by activating mitochondrial apoptotic signal pathway with up-regulated active-Caspase 3 and cytochrome-c protein expression.

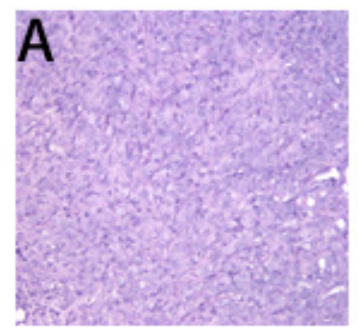

HE control

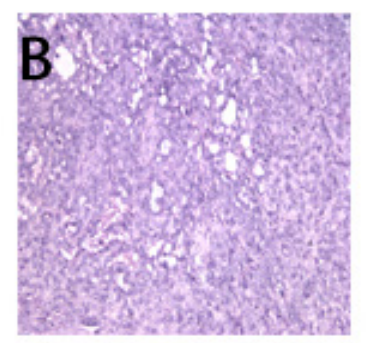

HE FAN

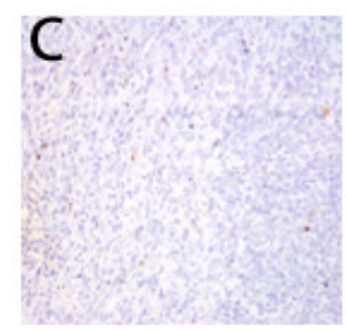

P27 control

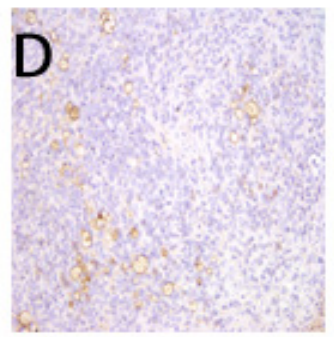

P27 FAN

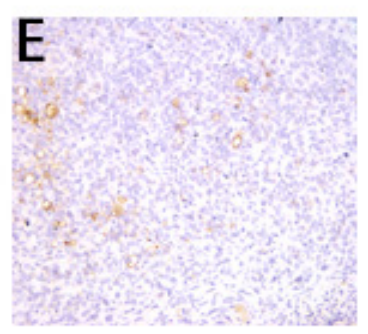

CD117 control

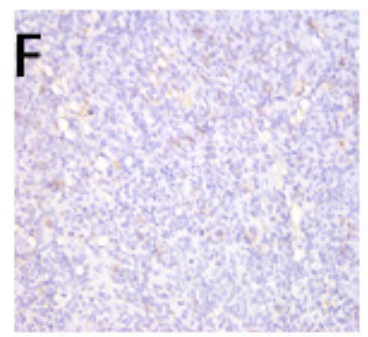

CD117 FAN

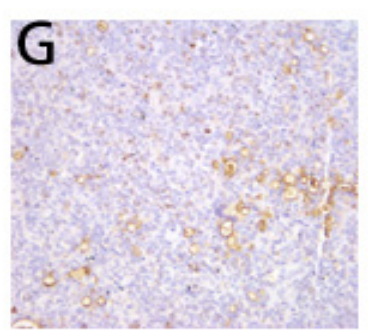

Ki-67 control

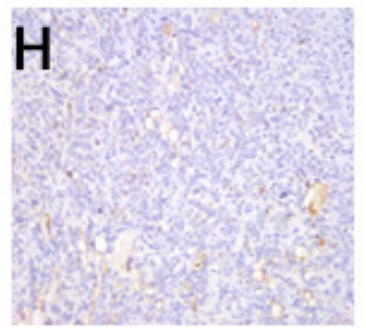

Ki-67 FAN

Figure 2: Fan increased P27 protein expression and repressed CD117 and Ki-67 protein expression in MDA-MB-231 cell xenograft. HE staining and immunohistochemistry staining (A, B). With $5 \mathrm{mg} / \mathrm{kg}$ Fan stimulation, p27 protein was induced in tumor(C, D). In contrary, with $5 \mathrm{mg} / \mathrm{kg}$ Fan stimulation, CD117 (E, F) and Ki-67(G, H) protein were reduced in tumor

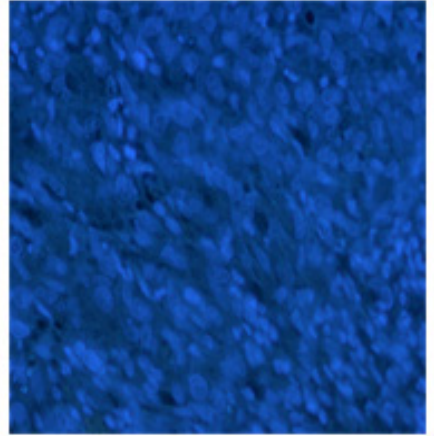

DAPI control

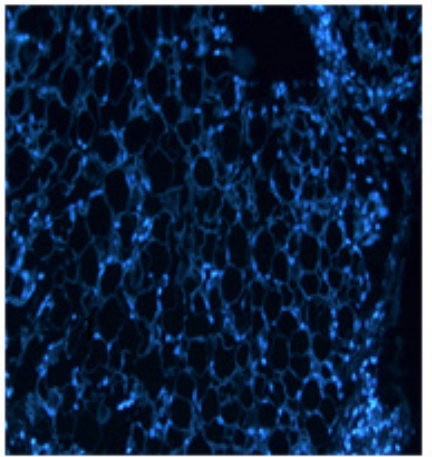

DAPI FAN

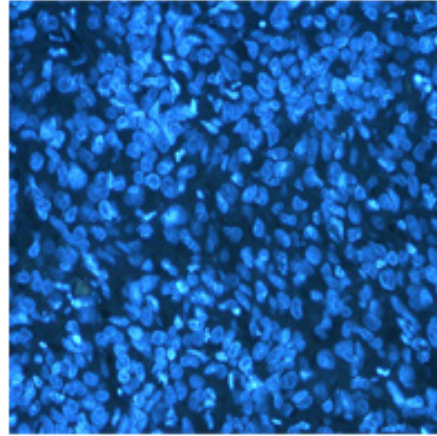

hochest control

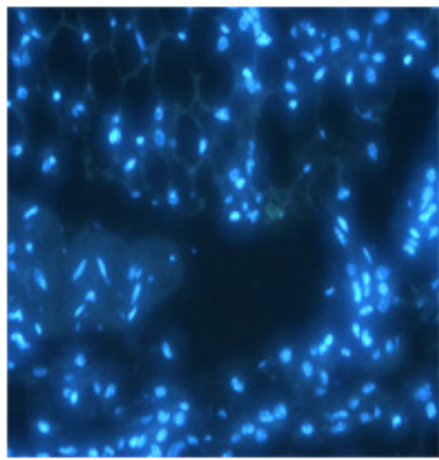

hochest FAN

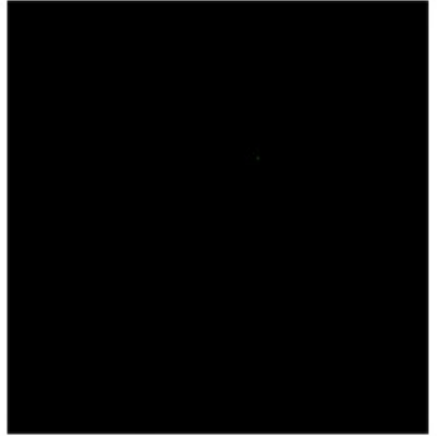

TUNEL (control)

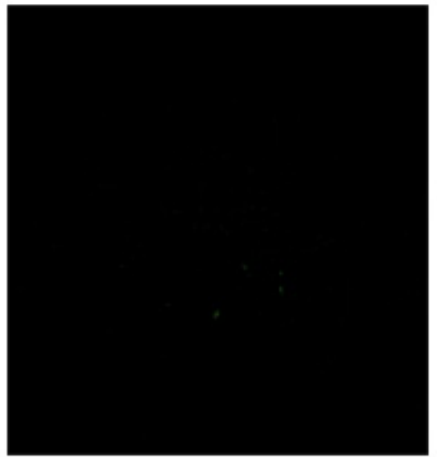

TUNEL (FAN)

Figure 3: Fan induced tumor apoptosis in MDA-MB-231 cell xenograft. DAPI staining. The nuclei of tumor cells were condensed and fragmented in the Fan treated tumor as indicated by the arrows, compared with the control tumor cells. Hoechst staining. The nuclei of tumor cells were condensed and fragmented in the Fan treated tumor as indicated by the arrows, compared with the control tumor cells. The TUNEL positive cells were indicated by the arrow compared with the control tumor cells 
A

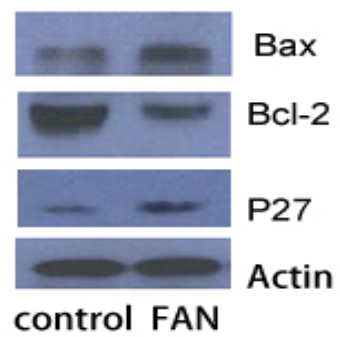

C

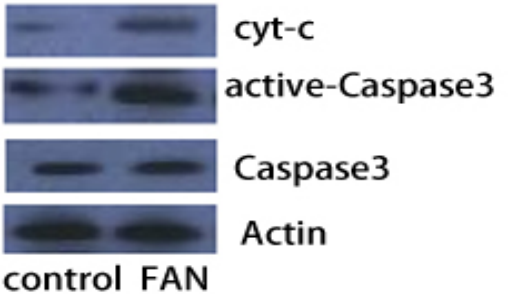

B
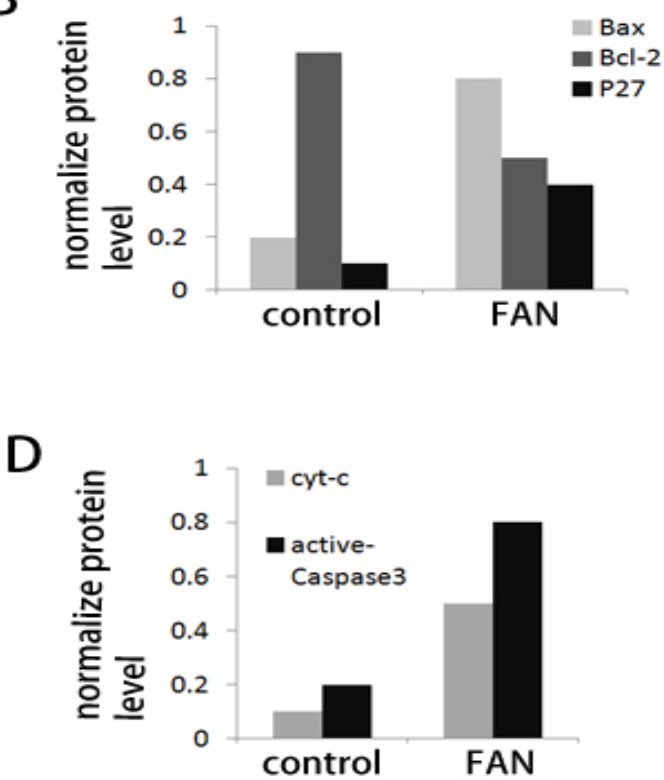

Figure 4: Fan induced tumor apoptosis via increasing Bax, p27, cytochrome-c and active-caspase3 protein expression and repressing Bcl-2 protein expression in MDA-MB-231 cell xenograft (A) Western blot of Bax, p27 and Bcl-2 protein in control and Fan (5mg/kg) treated mice for 16 days in MDA-MB-231 cell xenograft. For Bax and P27 proteins, the signals were strong in Fangchinoline (5mg/kg) stimulated for 16days in MDA-MB-231 cell xenograft. However, for Bcl-2 protein, the signals were stronger in control; (B) Quantification of Bax, p27 and Bcl-2 levels by western blot as in A. Bax, p27 and Bcl-2 protein levels in tumor were normalized to the loading control beta-actin. The level of Bax and p27 in the tumor treated with Fan (5mg/ $\mathrm{kg}$ ) was 4 and 5 times higher than that in the control respectively. The level of Bcl-2 in the tumor treated with Fan ( $5 \mathrm{mg} / \mathrm{kg})$ was 0.55 times lower than that in the control respectively; (C) Western blot of cytochrome-c, Caspase 3 and active-Caspase 3 protein in control and Fan (5mg/kg) treated mice for 16 days in MDA-MB-231 cell xenograft. For cytochrome-c and active-Caspase3 protein, the signals were strong in Fan (5mg/kg) treated group for 16 days in MDA-MB-231 cell xenograft and weak in control group. However, for Caspase3 protein, there was no significant difference in signals between control and Fan $(5 \mathrm{mg} / \mathrm{kg}$ ) stimulated group for 16days in MDA-MB-231 cell xenograft; (D) Quantification of cytochrome-c and active-Caspase3 levels by western blot as in C. Cytochrome-c and active-Caspase 3 protein levels in tumor were normalized to the loading control beta-actin. The level of cytochrome-c and active-Caspase 3 in the tumor treated with Fan $(5 \mathrm{mg} / \mathrm{kg})$ was 5 and 4 times higher than that in the control respectively

\section{Discussion}

Here, we demonstrated that Fan repressed the volume of breast cancer xenograft and induced apoptosis. Previously, Choi HSC et al. [4,5] reported that Fan has Anti-inflammatory effects. Zhang YH et al. [8,9] also reported that Fan inhibited rat aortic vascular smooth muscle cell proliferation and cell cycle progression through inhibition of ERK1/2 activation and c-fos expression. However, this is the first report to demonstrate that Fan induced apoptosis in breast cancer xenograft and Fan could be consider as a potential drug for future breast tumor therapy.

First, the tumor volume in xenograft treated by Fan was smaller than the control group (Figure 1). HE staining displayed that the tumor cell numbers treated by Fan are decreased compared with the control in Figure 2. These results demonstrated that Fan arrested tumor cell cycle or inhibited the cell proliferation. P27 is a member of the Cip/Kip family of cyclin-dependent kinase (Cdk) inhibitors that function to negatively regulate cyclin A-Cdk2 and cyclin E-Cdk2 complexes in the nucleus, preventing cell cycle progression [10]. We hypothesized that Fan could inhibit cell cycle of breast cancer cell and we tested the proteins expression of p27 by immunohistochemistry. And our results also showed that p27 protein expression was up-regulated by Fan in MDA-MB-231 breast tumor xenograft. CD117 antigen [11] and Ki67 [12] proteins are the marks of cell proliferation. Our results demonstrated that Fan down-regulated CD117 and Ki67 proteins expression compared with the control. Based on the results, we suggested that Fan down-regulated the marker proteins of CD117 and Ki67 and inhibited proliferation of MDA-MB-231 cells.

To identify if Fan induces breast cancer apoptosis, we applied DAPI and Hoechst staining and TUNEL immunofluorencese staining. DAPI and Hoechst staining results showed that the number of cell unclear decreased compared with the control group and the characteristics of apoptosis fragment and degradation of nuclear were found in the slides. TUNEL immunofluoresence staining result demonstrated that Fan up-regulated the positive cells of TUNEL compared with control. Mitochondrial outer-membrane permeabilization, which regulates the release of apoptosis promoting proteins from the mitochondrial intermembrane space, is mediated by pro and antiapoptotic members of the Bcl-2 family. The antiapoptotic family members Bcl-2 and Bclxl have been shown to block the release of apoptosis promoting proteins, whereas proapoptotic members such as Bax promote the apoptosis process. Therefore, we decided to explore the effect of Fan on Bax activation. Bax activation was investigated in tumor xenograft by western blot. Our result showed that Fan up-regulated Bax activation (Figure 4A). The change of mitochondrial membrane permeability is essential for apoptosis process. The decrease of mitochondrial membrane potential will lead to translocation of 
cytochrome $\mathrm{C}$ into cytoplasm. The Bcl-2 family proteins maintain the stability of mitochondrial membrane potential and the releasing of mitochondrial proteins by directly modulating the activity of voltage dependent anion channel. However, Bax alters the physical properties of the voltage dependent anion channel, making the voltage dependent anion channel permeable the cytochrome C, through its BH4 domain [13]. Fan up-regulated Bax activation in our experiment and this phenomena demonstrated that Bax increased the ability of voltage dependent anion channel permeable. The result also showed that cytochrome-C was upregulated by Fan. Since Bcl-2 is thought to protect cells from apoptosis by preventing the dissipation of voltage dependent anion channel and subsequent cytochrome-C release, we investigated the Bcl-2 protein expression level in Fan treated group. The result showed that Fan decreased the Bcl-2 protein level. From these results we found that Fan up-regualted Bax protein expression and down-regulated $\mathrm{Bcl}-2$ protein expression level, which induced cell apoptosis.

To further address the question if Fan directly causes the apoptosis, we tested protein expression levels of active caspase 3 using antibody against the active form of this protein. The result demonstrated that Fan up-regulated active Caspase 3 protein expression level. Many literatures reported that the apoptosis protein Bax and anti-apoptosis protein Bcl-2 are correlated proteins. Upregulation of active caspase 3 and cytochrome-c induces cell apoptosis [14-16]. Our results demonstrated that Fan up-regulated the ratio of Bax/Bcl-2 and also induced the protein expression levels of active Caspase 3 and cytochrome-c.

\section{Acknowledgement}

This work was supported by the Research Fund for the Doctoral Program of Higher Education of China (20125503120015 to Wang), Natural Science Foundation Project of CQ CSTC (cstc2014jcyjA10024 to Wang; 2011jjA10035 to Hu) and Research funding of Chongqing Medical University (Grant No.0800280031 to Wang).

\section{References}

1. Tang W, Eisenbrand G, Stephaniae tetrandrae S (1992) Moore Chinese Drugs of Plant Original, Springer-Verlag, Berlin, Germany.

2. Shen YC, Chen CF, Wang SY, Sung YJ (1999) Impediment to Calcium Influx and Reactive Oxygen Production Accounts for the Inhibition of Neutrophil Mac-1 Up-Regulation and Adhesion by Tetrandrine Mol pharmacol 55: 186-93.

3. Jen-Hao Hsu, Yang-Chang Wu, Shorong-Shii Liou, I-Min Liu, Lee-Wen Huang et al. (2009) Mediation of Endogenous endorphin by Tetrandrine to Lower Plasma Glucose in Streptozotocin-induced Diabetic Rats. Neurochem Int 54: 506-12.

4. Choi HS, Kim HS, Min KR, Kim Y, Lim HK, et al. (2000) Anti-inflammatory effects of fangchinoline and tetrandrine. J Ethnopharmacol 69: 173-9.

5. Gulci I, Elis R, Gepdiremen A, Chea A, Topal F (2010) Antioxidant activity of bisbenzylisoquinoline alkaloids from Stephania rotunda: cepharanthine and fangchinoline. J Enzyme Inhib Med Chem 25: 44-53.

6. Zhang YH, Fang LH, Ku BS (2003) Fangchinoline inhibits rat aortic vascular smooth muscle cell proliferation and cell cycle progression through inhibition of ERK1/2 activation and c-fos expression. Biochem Pharmacol 66: 1853-60.

7. Adriaenssens E, Vanhecke E, Saule P, Mougel A, Page A, et al. (2008) Nerve Growth Factor Is a Potential Therapeutic Target in Breast Cancer. Cancer Res 68: 346-51.

8. Ahn RW, Chen F, Chen H, Stern ST, Clogston JD, et al. (2010) A Novel Nanoparticulate Formulation of Arsenic Trioxide with Enhanced Therapeutic Efficacy in a Murine Model of Breast Cancer. Clin Cancer Res 16: 3607-17.

9. Challita-Eid PM, Morrison K, Etessami S, An Z, Morrison KJ, et al. (2007) Monoclonal Antibodies to Six-Transmembrane Epithelial Antigen of the Prostate-1 Inhibit Intercellular Communication In vitro and Growth of Human Tumor Xenografts In vivo. Cancer Res 67: 5798-805.

10. Short JD, Houston KD, Dere R, Cai SL, Kim J, et al. (2008) AMP-Activated Protein Kinase Signaling Results in Cytoplasmic Sequestration of p27. Cancer Res 68: 6496-506.

11. Cascavilla N, Musto P, D’Arena G, Melillo L, Carella AM,, et al. (1998) CD117 (c-kit) is a restricted antigen of acute myeloid leukemia and characterizes early differentiative levels of M5 FAB subtype. Haematologica 83: 392-7.

12. Noronha Y, Raza A, Hutchins B, Chase D, Garberoglio C, et al. (2011) CD34, CD117, and Ki-67 Expression in Phyllodes Tumor of the Breast: An Immunohistochemical Study of 33 Cases. Int J surg pathol 19: 152-8.

13. Bessert DA, Skoff RP (1999) High-resolution In Situ Hybridization and TUNEL Staining with Free-floating Brain Sections. J Histochem Cytochem 47: 693-702.

14. Katiyar SK, Roy AM, Baliga MS (2005) Silymarin induces apoptosis primarily through a p53-dependent pathway involving Bcl-2/Bax, cytochrome c release, and caspase activation. Mol Cancer Ther 4: 207-16.

15. Huang XF, Luo SK, Xu J, Li J, Xu DR, et al. (2008) Aurora kinase inhibitory VX-680 increases Bax/Bcl-2 ratio and induces apoptosis in Aurora-A-high acute myeloid leukemia. Blood 111: 2854-65.

16. Mantena SK, Sharma SD, Katiyar SK (2006) Berberine, a natural product, induces G1-phase cell cycle arrest and caspase-3-dependent apoptosis in human prostate carcinoma cells. Mol Cancer Ther 5: 296-308. 


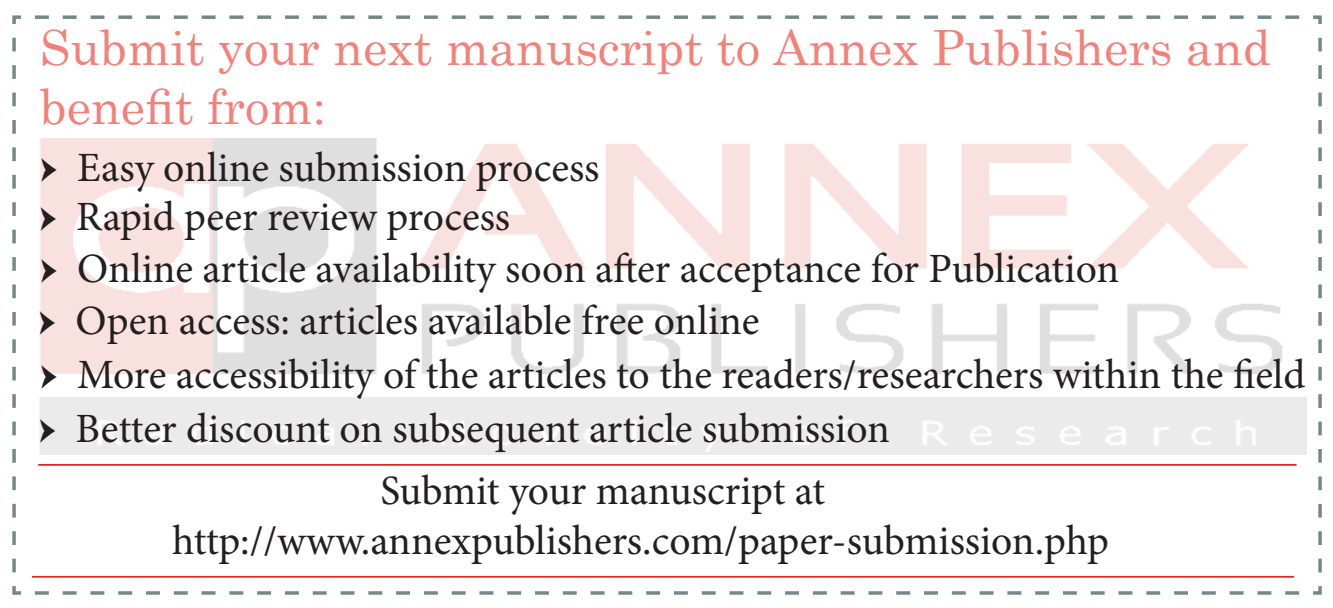

